Article

\title{
Numerical Study of Natural Convection Flow of Nanofluid Past a Circular Cone with Cattaneo-Christov Heat and Mass Flux Models
}

\author{
Muhammad Saleem Iqbal ${ }^{1}$, Waqar Khan ${ }^{2}$, Irfan Mustafa $^{3}$ and Abuzar Ghaffari ${ }^{4, *(\mathbb{D}}$ \\ 1 Department of Mathematics, Islamabad College for Boys G-6/3, Islamabad 44000, Pakistan; \\ saleem366@yahoo.com \\ 2 Department of Mechanical Engineering, College of Engineering, Prince Mohammad Bin Fahd University, \\ Al Khobar 31952, Saudi Arabia; wkhan1956@gmail.com \\ 3 Department of Mathematics, Allama Iqbal Open University, H-8, Islamabad 44000, Pakistan; \\ irfan.mustafa@aiou.edu.pk \\ 4 Department of Mathematics, University of Education, Lahore (Attock Campus) 43600, Pakistan \\ * Correspondence: abuzar.ghaffari@ue.edu.pk
}

Received: 24 July 2019; Accepted: 23 August 2019; Published: 3 November 2019

\begin{abstract}
The objective of this study is to analyze the natural convection flow of nanofluid along a circular cone placed in a vertical direction. The generalized heat flux and mass flux models are commonly known as the Cattaneo-Christov heat flux model and mass flux models. In the present study, these models are used for both heat and mass transfers analysis in nanofluid flow. For the governing equations, the Buongiorno transport model is used in which two important slip mechanism, namely thermophoresis and Brownian motion parameters, are discussed. The resulting governing equations in the form of partial differential equations (PDEs) are converted into ordinary differential equations (ODEs) due to similar flow along the surface of a circular cone. To solve these ODEs, a numerical algorithm based on implicit finite difference scheme is utilized. The effects of dimensionless parameters on heat and mass transfer in nanofluid flow are discussed graphically in the form of velocity profile, temperature profile, Sherwood number and Nusselt number. It is noted that in the presence of the Cattaneo-Christov heat flux model and mass flux model, the heat transfer rate decreases by increasing both thermal and concentration relaxation parameters; however, Sherwood number decreases by increasing the thermal relaxation parameter, and increases by increasing the concentration relaxation parameter.
\end{abstract}

Keywords: Cattaneo-Christov model; Buongiorno's transport model; circular cone; natural convection flow

\section{Introduction}

Improvement in the efficiency of cooling systems is essential in various industrial and engineering processes. The excellent functioning of a cooling system requires a thermally efficient coolant. However, the relatively poor thermal conductivity of conventional coolants is a significant constraint on the emergence of highly effective cooling systems. In most of the heat transfer processes, conventional fluids or base fluids like water, engine oil, and ethylene glycol, etc. are used as coolants. Due to low thermal conductivity of these fluids, they do not provide efficient results in a cooling system. An inventive procedure for enhancing the heat transfer rate in the conventional fluids is by colloidal suspension of nanosized particles in base fluid, resulting in a mixture known as nanofluid [1,2]. Nanofluid exhibits excellent potential, considering the substantial increase in heat transfer rates in a variety of applications. Nanotechnology has been the source for the creation of nanoparticles in dimensions of nanometers, 
which possess distinctive chemical and physical properties. Nanofluid possesses high thermal conductivity and promotes the heat transfer rate. Nanofluid helps in manufacturing light and smart heat exchangers. Due to nano-sized particles, the fluid mixture is homogenous and stable without having the problems of sedimentation and clogging. Nanofluids are most suitable for heating and cooling systems. Therefore, for heating, nanofluids can be used to improve the heat transfer phenomena in diverse thermal systems; and for cooling, its applications include engine cooling, refrigeration, use in the petroleum industry, cancer therapy, nano-drug delivery, environmental remediation, inkjet printing, etc. The work of the nanofluid fundamentally depends upon the size, percentage of nanoparticle concentration, ability to stay suspended in base fluid, shape, and chemical unreactive in the base fluid.

Heat transfer is an important phenomenon in nature, which occurs due to the temperature difference between two bodies or within the same body. The heat transfer characteristics have wide-ranging demands in numerous industrial and engineering processes, such as: nuclear reactors, fuel cells, transportation, microelectronics, etc. Fourier's law of heat conduction explains the heat transfer phenomena in various practical processes. Fourier's law gives a parabolic energy equation in which the whole system is instantly affected by the initial disturbance. To handle this situation, a modified version of Fourier's heat conduction law was introduced by Cattaneo including a relaxation time. Christov incorporated Cattaneo's theory and replaced the time derivative with an Oldroyd upper-convected derivative. The derivative model of Cattaneo's law is termed as the Cattaneo-Christov heat flux model.

Many researchers and scientists studied self-similar solutions for natural convection flow over a vertical cone. Hering and Grosh [3,4] studied natural convection flow along the vertical cone and reported the similarity solution. Roy [5] investigated the heat transfer phenomena for a large Prandtl number in natural convection flow along the vertical cone. Vajravelu and Nayfeh [6] studied heat transfer analysis in a viscous heat source fluid along a cone and wedge surface and concluded that the flow and heat transfer rates have smaller values along the cone surface, as compared to the wedge surface. The study of Kafoussias [7] is related to the isothermal vertical cone. He grasped from the results, that bouncy parameter and Schmidt number strongly affect the heat and mass transfer rates.

Yih [8] studied the radiation effects along a truncated isothermal cone and found the significant contribution in the enhancement of Nusselt numbers. Behrang et al. [9] performed heat transfer analysis along a vertical cone saturated in a porous medium. He established a new approach named the hybrid neural network-particle swarm optimization method and concluded that values of the Nusselt numbers found a good agreement with the numerically computed values. Cheng [10] discussed natural convection flow of Newtonian fluid along the non-isothermal permeable vertical cone with suction and variable properties. Through results, he concluded that the Nusselt number becomes high with the increase of suction and viscosity variation parameters. Duwairi et al. [11] considered magnetohydrodynamic (MHD) mixed convection flow over a cone and observed that the Nusselt number increases with the increase of cone angle in a porous medium. Elbashbeshy et al. [12] examined natural convection flow phenomena along a vertical circular cone in the presence of pressure work, variable heat flux and heat generation. They observed that the skin friction increases, and the Nusselt number decreases with the increments in heat generation parameters. Braun et al. [13] studied free convection similarity flows along the families of bodies with closed lower ends and observed that the body shape parameter enhances the heat transfer rate. Grosan [14] examined free convection flow over a vertical cone in a viscoelastic fluid with a heat source in a porous medium. Chamkha et al. [15] discussed the effects of a combined chemical reaction and pressure work in natural convection flow. They found that the Nusselt number decayed with the enhancement of heat generation, chemical reaction parameter, and the Schmidt number. Sohouli et al. [16] analyzed the free convection analytically by using the Homotopy Analysis Method (HAM) in the Darcian fluid along a vertical cone.

Several researchers and scientists examined different techniques and models to explain the heat transfer phenomena of nanofluid. There are two types of models available in the literature, namely, the homogenous and the non-homogenous. Among homogenous theoretical models, the Buongiorno 
transport model $[17,18]$ and Tiwari and Das model [19] are the most famous ones. Buongiorno (2006) developed a theoretical model for convective transport in nanofluid by incorporating Brownian motion and thermophoresis effects, whereas thermophysical properties of the nanoparticles were introduced by Tiwari and Das [19] in their proposed model.

In the recent past, attention has been given to the boundary layer flow of nanofluid over a cone. Mahdy [20] numerically computed the Sherwood and Nusselt numbers for the case of natural convection flow along a vertical cone. He noticed with the variation of Brownian motion and thermophoresis parameters, the Nusselt number decreased and the Sherwood number increased. Behseresht et al. [21] discussed the free convection flow of nanofluid along a vertical cone using the Buongiorno model in a porous medium. They noticed that the change in the heat transfer rate is negligible due to the migration of nanoparticles in comparison with convection and heat conduction phenomenon. Noghrehabadi et al. [22] discussed the natural convection flow of a nanofluid past an isothermal vertical cone in a non-Darcy porous medium and noted that both heat and mass transfer rates reduce with increasing non-Darcy parameters. Keshtkar and Hadizadeh [23] investigated boundary layer nanofluid flow along a vertical cone in a porous medium. Fauzi et al. [24] studied mixed convection nanofluid flow along a vertical cone. In a series of papers, Khan et al. $[25,26]$ presented mathematical models to investigate the natural convection flow of a water-based nanofluid containing gyrotactic microorganisms over a truncated cone with a convective boundary condition at the surface. They found that in case of non-Newtonian nanofluids, the local Nusselt and the local Sherwood numbers are found to be higher for dilatant nanofluids than pseudoplastic and Newtonian fluid.

Straughan [27] investigated thermal convection phenomena with the Cattaneo-Christov heat flux model. Tibulle and Zampoli [28] examined the Cattaneo-Christov heat flux model for incompressible fluid flows. Kumar et al. [29] studied the MHD flow over a cone and a wedge with the Cattaneo-Christov heat flux model and shows that the heat transfer rate in the fluid flow over a cone is higher than that of the flow over a wedge. Further, numerous researchers [30-35] used the Cattaneo-Christov heat flux model to formulate energy equations and discuss the flow and heat transfer phenomenon for different types of non-Newtonian fluids.

With such an intensive literature review, we came to know that a study on natural convection flow along a vertical cone under the effects of Brownian motion, thermophoresis parameter, buoyancy force and the presence of Cattaneo-Christov heat flux model, has not yet been examined. To deal with this theoretical investigation, self-similar transformations are used and obtained by a coupled system of non-linear ordinary differential equations. The problem is solved numerically by applying the Keller-box scheme [36,37]. The impact of different involved parameters on the concentration, temperature and velocity profiles, Nusselt number, Sherwood number and skin friction are presented through graphs. The numerical values of the Nusselt and the Sherwood numbers are computed and reported in the form of Tables.

\section{Mathematical Formulation}

Steady two-dimension flow problem along a vertical cone of a circular base with radius $r$ is considered. The symbol $\gamma$ is used to represent the internal half-angle cone. The temperature and concentration at the surface of the circular cone are kept constant, $T_{w}$ i.e., $\left(T_{w}>T_{\infty}\right)$ and $C_{w}\left(C_{w}>C_{\infty}\right)$. The symbols $T_{\infty}$ and $C_{\infty}$ are used to represent the constant ambient temperature and concentration far away from the surface. The flow is developed in an upward direction. The $x$-axis is taken along the surface of the cone, and the $y$-axis is taken normal to it as depicted in Figure 1. 


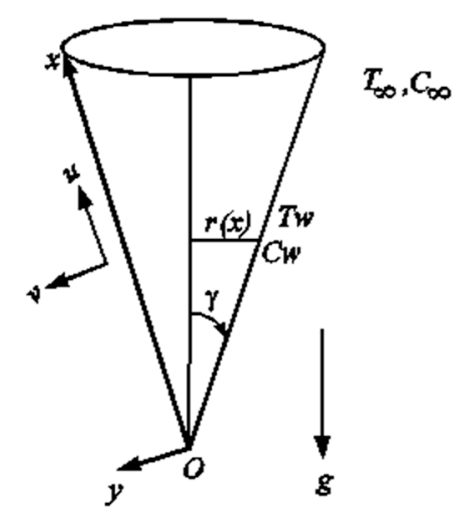

Figure 1. Flow geometry and the coordinate system.

Brownian motion and thermophoresis effects of nanoparticles are considered, which are studied using the Buongiorno nanofluid model. According to the Buongiorno's model [21,22], the mass, momentum and energy conservation laws after the consideration of the above assumptions are written as:

Mass

$$
\frac{\partial(\overline{r u})}{\partial \bar{x}}+\frac{\partial(\overline{r v})}{\partial \bar{y}}=0
$$

Momentum

$$
\bar{u} \frac{\partial \bar{u}}{\partial \bar{x}}+\bar{v} \frac{\partial \bar{u}}{\partial \bar{y}}=v \frac{\partial^{2} \bar{u}}{\partial \bar{y}^{2}}+g \beta\left(T-T_{\infty}\right) .
$$

The generalized heat and mass flux models for the thermal and concentration diffusions are commonly termed as Cattaneo-Christov heat and mass flux models. These models are defined as:

$$
\begin{gathered}
\boldsymbol{q}+\lambda_{E}\left(\frac{\partial q}{\partial t}+\boldsymbol{V} \cdot \nabla \boldsymbol{q}-\boldsymbol{q} . \nabla \boldsymbol{V}+(\nabla \cdot \boldsymbol{V}) \boldsymbol{q}\right)=-\kappa \nabla T, \\
J+\lambda_{C}\left(\frac{\partial J}{\partial t}+\boldsymbol{V} . \nabla \boldsymbol{J}-\boldsymbol{J} . \nabla \boldsymbol{V}+(\nabla . \boldsymbol{V}) \boldsymbol{J}\right)=-D_{m} \nabla C,
\end{gathered}
$$

where $\lambda_{E}$ and $\lambda_{C}$ are the relaxation time parameter for heat flux and mass flux, $k$ represents the thermal conductivity, and $D_{m}$ is used to represent the mass diffusion coefficient. The above-generalized flux models become to Fourie and Fick's laws, if $\lambda_{E}=0$, and $\lambda_{C}=0$, that is:

$$
\begin{gathered}
q+\lambda_{E}(\boldsymbol{V} . \nabla \boldsymbol{q}-\boldsymbol{q} . \nabla \boldsymbol{V})=-\kappa \nabla T, \\
J+\lambda_{C}(\boldsymbol{V} . \nabla \boldsymbol{J}-\boldsymbol{J} . \nabla \boldsymbol{V})=-D_{m} \nabla C .
\end{gathered}
$$

Hence after the incorporation of Cattaneo-Christov heat and mass flux models, energy and concentration equations can be written as $[19,20]$ :

Energy

$$
\bar{u} \frac{\partial T}{\partial \bar{x}}+\bar{v} \frac{\partial T}{\partial \bar{y}}+\lambda_{E} \phi_{E}=\alpha \frac{\partial^{2} T}{\partial \bar{y}^{2}}+\tau\left(D_{B} \frac{\partial T}{\partial \bar{y}} \frac{\partial C}{\partial \bar{y}}+\frac{D_{T}}{T_{\infty}}\left(\frac{\partial T}{\partial \bar{y}}\right)^{2}\right),
$$

Concentration

$$
\bar{u} \frac{\partial C}{\partial \bar{x}}+\bar{v} \frac{\partial C}{\partial \bar{y}}+\lambda_{C} \phi_{C}=D_{B} \frac{\partial^{2} T}{\partial \bar{y}^{2}}+\frac{D_{T}}{T_{\infty}} \frac{\partial^{2} T}{\partial \bar{y}^{2}},
$$

where

$$
\phi_{E}=\left(\left(\bar{u} \frac{\partial \bar{u}}{\partial \bar{x}}+\bar{v} \frac{\partial \bar{u}}{\partial \bar{y}}\right) \frac{\partial T}{\partial \bar{x}}+\left(\bar{u} \frac{\partial \bar{v}}{\partial \bar{x}}+v \frac{\partial \bar{v}}{\partial \bar{y}}\right) \frac{\partial T}{\partial \bar{y}}+2 \overline{u v} \frac{\partial^{2} T}{\partial \bar{x} \partial \bar{y}}+\bar{u}^{2} \frac{\partial^{2} T}{\partial \bar{x}^{2}}+\bar{v}^{2} \frac{\partial^{2} T}{\partial \bar{y}^{2}}\right) .
$$


and

$$
\phi_{C}=\left(\left(\bar{u} \frac{\partial \bar{u}}{\partial \bar{x}}+\bar{v} \frac{\partial \bar{u}}{\partial \bar{y}}\right) \frac{\partial C}{\partial \bar{x}}+\left(\bar{u} \frac{\partial \bar{v}}{\partial \bar{x}}+v \frac{\partial \bar{v}}{\partial \bar{y}}\right) \frac{\partial C}{\partial \bar{y}}+2 \overline{u v} \frac{\partial^{2} C}{\partial \bar{x} \partial \bar{y}}+\bar{u}^{2} \frac{\partial^{2} C}{\partial \bar{x}^{2}}+\bar{v}^{2} \frac{\partial^{2} C}{\partial \bar{y}^{2}}\right) .
$$

The used symbols are defined as:

$\begin{array}{llll}D_{T}: & \text { Thermophoretic diffusion coefficient; } & \alpha: & \text { Thermal diffusivity; } \\ D_{B}: & \text { Brownian diffusion coefficient; } & \mathrm{g}: & \text { Gravitational acceleration; } \\ \tau: & \text { The ratio of heat capacity of a } & \mathrm{C} & \text { Concentration; }\end{array}$

$\tau$ : The ratio of heat capacity of a

nanoparticle to the base fluid;

$T$ Temperature;

The local radius $r$ of the cone surface is described as $\bar{r}(\bar{x})=\bar{x} \operatorname{Sin} \gamma$, as shown in Figure 1 . The appropriate boundary conditions are written as:

$$
\bar{y}=0: \bar{u}=0, \bar{v}=0, C=C_{w}, T=T_{w}, \bar{y} \rightarrow \infty: \bar{u}=0, \quad C=C_{\infty}, T=T_{\infty} .
$$

The stream function in the polar form is defined as $r v=-\partial \psi / \partial x, r u=\partial \psi / \partial y$, which satisfies the continuity equation identically. To achieve the dimensionless form, the following transformations are utilized (see [6]):

$$
\begin{aligned}
& \xi=x=\frac{\bar{x}}{l}, r=\frac{\bar{r}}{l}, \eta=\xi^{-\frac{1}{4}} \frac{\bar{y}}{l} G r^{\frac{1}{4}}, u=\frac{\rho l}{\mu} G r^{-\frac{1}{2}} \bar{u}, v=\frac{\rho l}{\mu} G r^{-\frac{1}{4}}(\bar{v}), \\
& r(\xi)=\xi \operatorname{Sin} \gamma, \operatorname{Pr}=\frac{v}{\alpha}, \varphi(\eta)=\frac{C-C_{\infty}}{C_{w}-C_{\infty}}, \theta(\eta)=\frac{T-T_{\infty}}{T_{w}-T_{\infty}}, \psi(\eta)=\xi^{\frac{3}{4}} r f(\eta),
\end{aligned}
$$

which transforms the governing PDEs (2)-(8) to dimensionless ODEs i.e.,

$$
\begin{gathered}
f^{\prime \prime \prime}+\frac{7}{4} f f^{\prime \prime}-\frac{1}{2} f^{\prime 2}+\theta=0, \\
\frac{1}{\operatorname{Pr}} \theta^{\prime \prime}+\frac{7}{4} f \theta^{\prime}-\delta_{1}\left(\left(\frac{35}{8}\right) f f^{\prime} \theta^{\prime}+\left(\frac{49}{16}\right) f^{2} \theta^{\prime \prime}\right)+N b \theta^{\prime} \varphi^{\prime}+N t \varphi^{\prime 2}=0, \\
\frac{1}{L e}\left(\varphi^{\prime \prime}+\frac{N t}{N b} \theta^{\prime \prime}\right)+\frac{7}{4} f \varphi^{\prime}-\delta_{2}\left(\left(\frac{35}{8}\right) f f^{\prime} \varphi^{\prime}+\left(\frac{49}{16}\right) f^{2} \varphi^{\prime \prime}\right)=0,
\end{gathered}
$$

where

$$
\begin{gathered}
G r=\frac{g \beta\left(T_{w}-T_{\infty}\right) l^{3}}{v^{2}}, L e=\frac{v}{D_{B}}, N b=\frac{\tau D_{B}\left(T_{w}-T_{\infty}\right)}{v}, N t=\frac{\tau D_{T}\left(C_{w}-C_{\infty}\right)}{T_{\infty} v}, \\
\operatorname{Pr}=\frac{v}{\alpha}, \quad \delta_{1}=\lambda_{E} v / l^{2} \sqrt{G r / x}, \delta_{2}=\lambda_{C} v / l^{2} \sqrt{G r / x}
\end{gathered}
$$

are the Grashof number, Lewis number, Brownian motion parameter, thermophoresis parameter, and Prandtl number respectively. Accordingly, the boundary conditions are also transformed and written as

$$
\begin{gathered}
f(0)=0, f^{\prime}(0)=0, \varphi(0)=1, \theta(0)=1, \\
f^{\prime}(\infty) \rightarrow 0, \varphi(\infty) \rightarrow 0, \theta(\infty) \rightarrow 0 .
\end{gathered}
$$

The aforementioned prime symbol " ' " is used to represent the differentiation with respect to $\eta$. The wall shear stress $\left(\tau_{w}=\mu(\nabla \bar{u})_{y=0}\right.$, ) the surface heat flux $\left(q_{w}=-\kappa(\nabla T)_{y=0}\right)$ and the surface mass flux $\left(q_{m}=-D_{B}(\nabla C)_{y=0}\right)$ are the quantities of physical interest. The coefficient of skin friction, the Nusselt number and the Sherwood number in the non-dimensional form are

$$
\begin{gathered}
C_{f}=C_{f x}(G r / x)^{\frac{1}{4}}=f^{\prime \prime}(0), \\
N u=-N u_{x}\left(G r x^{3}\right)^{-\frac{1}{4}}=-\theta^{\prime}(0), S h=-S h_{x}\left(G r x^{3}\right)^{-\frac{1}{4}}=-\varphi^{\prime}(0),
\end{gathered}
$$

where $C_{f x}=\tau_{w} / \rho U^{2}, N u_{x}=\bar{x} q_{w} / \kappa\left(T_{w}-T_{\infty}\right)$ and $S h_{x}=\bar{x} q_{m} / D_{B}\left(C_{w}-C_{\infty}\right)$ denote the coefficient of skin friction, the Nusselt number, and the Sherwood number respectively. 


\section{Method of Solution}

The Keller-box [36,37] method, based on the finite difference scheme, is used to find the solution in numerical form for Equations (13)-(15) with the given boundary conditions (Equation (17)). The implementation of the Keller-box method can be described in the following steps:

(i) The higher-order differential equations are transformed into the first-order. For this purpose, lets us consider

$$
f^{\prime}=U, f^{\prime \prime}=V, \theta^{\prime}=P, \phi^{\prime}=Q,
$$

then Equations (13)-(15) can take the following form

$$
\begin{gathered}
V^{\prime}+\frac{7}{4} f V-\frac{1}{2} U^{2}+\theta=0 \\
V^{\prime}+\frac{7}{4} f V-\frac{1}{2} U^{2}+\theta=0 \\
\frac{1}{P r} P^{\prime}+\frac{7}{4} f P-\delta_{1}\left(\frac{35}{8} f U P+\frac{49}{16} f^{2} P^{\prime}\right)+N_{b} P Q+N_{t} Q^{2}=0 \\
\frac{1}{L e}\left(Q^{\prime}+\frac{N t}{N b} P^{\prime}\right)+\frac{7}{4} f Q-\delta_{2}\left(\left(\frac{35}{8}\right) f U Q+\left(\frac{49}{16}\right) f^{2} Q^{\prime}\right)=0,
\end{gathered}
$$

and the boundary conditions are

$$
\begin{array}{r}
f=0, \quad U=0, \quad \theta=1, \quad \phi=1 \quad \text { at } \quad \eta=0, \\
f=0, \quad U=0, \quad \theta=1, \quad \phi=1 \quad \text { at } \quad \eta=0, \\
U=0, \quad \theta=0, \quad \phi=0 \quad \text { as } \quad \eta \rightarrow \infty .
\end{array}
$$

(ii) The derivatives are discretized by the central difference formula

$$
()_{j-\frac{1}{2}}=\frac{1}{h}\left(()_{j}-()_{j-1}\right)
$$

and the functions are replaced by their means value like

$$
()_{j-\frac{1}{2}}=\frac{1}{2}\left(()_{j}+()_{j-1}\right)
$$

(iii) The discretized nonlinear algebraic equations are linearized with the help of Newton's technique. For this purpose, the functions at $(i+1)$ th iteration are written as

$$
()_{i+1}=()_{i}+\delta()_{i}
$$

where $\delta()_{i}$ represents the small increment in the function's values. The second and higher orders' terms in $\delta()_{i}$ are neglected.

(iv) Linearized algebraic equations are finally solved through block-tridiagonal elimination method.

The present results of the skin friction coefficient $C_{f x}(G r / x)^{\frac{1}{4}}$ and heat transfer rate $N u_{x}\left(G r x^{3}\right)^{-1 / 4}$ have been compared with the available results of Yih et al. [8] in Table 1, and excellent agreement is achieved. This validates our current solution scheme and allows for further analysis. 
Table 1. Comparison of the current solution with the already existing results.

\begin{tabular}{ccccc}
\hline \multirow{2}{*}{$\boldsymbol{P r}$} & \multicolumn{2}{c}{$f^{\prime}(0,0)$} & \multicolumn{2}{c}{$-\boldsymbol{\theta}^{\prime}(0,0)$} \\
\cline { 2 - 5 } & Present & Yih et al. [8] & Present & Yih et al. [8] \\
\hline 0.0001 & 1.452616 & 1.6006 & 0.033845 & 0.0079 \\
0.001 & 1.440436 & 1.5135 & 0.038402 & 0.0246 \\
0.01 & 1.348483 & 1.3551 & 0.075460 & 0.0749 \\
0.1 & 1.095916 & 1.0960 & 0.211345 & 0.2116 \\
0.7 & 0.819591 & - & 0.451095 & - \\
1 & 0.769428 & 0.7699 & 0.510399 & 0.5109 \\
10 & 0.487697 & 0.4877 & 1.033989 & 1.0339 \\
100 & 0.289635 & 0.2896 & 1.922854 & 1.9226 \\
1000 & 0.166145 & 0.1661 & 3.470171 & 3.4696 \\
10000 & 0.094042 & 0.0940 & 6.200679 & 6.1984 \\
\hline
\end{tabular}

\section{Results and Discussion}

The graphical representations of the dimensionless velocity $f^{\prime}(\eta)$, temperature $\theta(\eta)$, and nanoparticle concentration $\varphi(\eta)$ profiles, in addition to the local Sherwood number $S h=-\varphi^{\prime}(0)$ and local Nusselt number $N u==-\theta^{\prime}(0)$ are made in this Section. The impact of the involved parameters on the graphs of the quantities named above are discussed in detail. Some numerical values of $S h$ and $N u$ against various values of parameters $\gamma_{1}, \gamma_{2}, N b, L e, N t$, and $P r$ are given in Tables 2-4. Table 2 reveals that the $N u$ reduces with the increase of $N t$ and $N b$; however, $S h$ rises with increasing $N t$ and $N b$. Table 2 shows that $S h$ decreases for high values of $N t$ and $N b$. Table 3 provides numerical results for the impact of $\mathrm{Pr}$ and $L e$ on heat transfer rate $\mathrm{N} u$ and mass transfer rate $S h$. It is noted that $\mathrm{Nu}$ decreases and $S h$ increases with increasing $P r$ and Le. Table 4 is prepared for the numerical data of the local Nusselt number $N u$ and Sherwood number $S h$ for various values of the thermal relaxation parameter $\delta_{1}$ and concentration relaxation parameter $\delta_{2}$. It is observed that $N u$ has lower values when the larger values of $\delta_{1}$ and $\delta_{2}$ are taken. It is further observed that numerical values of $S h$ are higher for increasing values of $\delta_{2}$ and lower for higher values of $\delta_{1}$.

Table 2. The $N u$ and $S h$ values for different $N t$ and $N b$ when $L e=P r=1.0, \delta_{1}=\delta_{2}=0.1$.

\begin{tabular}{|c|c|c|c|c|c|c|c|c|c|c|}
\hline \multirow{2}{*}{$\mathrm{Nt} / \mathrm{Nb}$} & \multicolumn{2}{|c|}{0.1} & \multicolumn{2}{|c|}{0.2} & \multicolumn{2}{|c|}{0.3} & \multicolumn{2}{|c|}{0.4} & \multicolumn{2}{|c|}{0.5} \\
\hline & $N u$ & $S h$ & $N u$ & $S h$ & $N u$ & Sh & $N u$ & $S h$ & $N u$ & $S h$ \\
\hline 0.1 & 0.47256 & 0.29844 & 0.45902 & 0.10786 & 0.44588 & -0.06091 & 0.43312 & -0.2091 & 0.42074 & -0.33788 \\
\hline 0.2 & 0.45094 & 0.41891 & 0.43785 & 0.33380 & 0.42515 & 0.25910 & 0.41284 & 0.19421 & 0.40089 & 0.13854 \\
\hline 0.3 & 0.42988 & 0.46077 & 0.41724 & 0.4106 & 0.40499 & 0.36703 & 0.39311 & 0.32968 & 0.38161 & 0.29816 \\
\hline 0.4 & 0.40938 & 0.48295 & 0.39720 & 0.45007 & 0.38540 & 0.42190 & 0.37396 & 0.39814 & 0.36290 & 0.37853 \\
\hline 0.5 & 0.38947 & 0.49724 & 0.37774 & 0.47459 & 0.36639 & 0.45551 & 0.3554 & 0.43978 & 0.34476 & 0.42718 \\
\hline
\end{tabular}

Table 3. $N u$ and $S h$ data for different $P r$ and $L e$ when $N t=N b=\delta_{1}=\delta_{2}=0.1$.

\begin{tabular}{ccccccc}
\hline \multirow{2}{*}{$\boldsymbol{P r} /$ Le } & \multicolumn{2}{c}{$\mathbf{2}$} & \multicolumn{2}{c}{$\mathbf{3}$} & \multicolumn{2}{c}{5} \\
\cline { 2 - 7 } & $\mathbf{N u}$ & $\mathrm{Sh}$ & $\mathbf{N u}$ & $\mathrm{Sh}$ & $\mathrm{Nu}$ & $\boldsymbol{S h}$ \\
\hline 2 & 0.54899 & 0.44100 & 0.59205 & 0.37685 & 0.63310 & 0.30480 \\
3 & 0.53896 & 0.61442 & 0.57534 & 0.56004 & 0.6035 & 0.50459 \\
5 & 0.52795 & 0.84874 & 0.55651 & 0.80533 & 0.5693 & 0.76937 \\
\hline
\end{tabular}


Table 4. $N u$ and $S h$ values for different $\gamma_{1}$ and $\gamma_{2}$ when $N t=N b=0.1, P r=L e=1.0$.

\begin{tabular}{ccccccccc}
\hline \multirow{2}{*}{$\boldsymbol{\delta}_{1} \rightarrow / \delta_{2} \downarrow$} & \multicolumn{2}{c}{$\mathbf{0 . 0}$} & \multicolumn{2}{c}{$\mathbf{0 . 2}$} & \multicolumn{2}{c}{$\mathbf{0 . 3}$} & \multicolumn{2}{c}{$\mathbf{0 . 4}$} \\
\cline { 2 - 9 } & $\boldsymbol{N u}$ & $\boldsymbol{S h}$ & $\boldsymbol{N u}$ & $\boldsymbol{S h}$ & $\boldsymbol{N u}$ & $\boldsymbol{S h}$ & $\mathbf{N u}$ & $\boldsymbol{S h}$ \\
\hline 0.0 & 0.47432 & 0.29986 & 0.47140 & 0.29258 & 0.47015 & 0.28898 & 0.46904 & 0.28545 \\
0.2 & 0.47384 & 0.30440 & 0.47091 & 0.29714 & 0.46966 & 0.29351 & 0.46855 & 0.28992 \\
0.3 & 0.47358 & 0.30685 & 0.47064 & 0.29961 & 0.46939 & 0.29597 & 0.46827 & 0.29235 \\
0.4 & 0.47331 & 0.30945 & 0.47036 & 0.30221 & 0.46910 & 0.29857 & 0.46798 & 0.29493 \\
\hline
\end{tabular}

Figure 2 illustrates the impact of thermal and concentration relaxation parameters $\delta_{1}$ and $\delta_{2}$ on the dimensionless profiles i.e., $(\eta), \theta(\eta)$, and $\varphi(\eta)$. It is observed that the thermal relaxation parameter reduces the velocity profile, whereas no significant change occurs in the case of the concentration relaxation parameter. It is further observed the temperature within the nanofluid reduces with increasing the values of $\delta_{1}$, but enhancement is observed in the dimensionless concentration profile. Figure 2 also shows that $\delta_{2}$ temperature profile increases whereas concentration distribution decreases. Additionally, reduction in the concentration profile is more prominent, as compared to enhancement in the temperature distribution. Overall, temperature and concentration distributions within nanofluid become higher in the case of $\delta_{2}$ and $\delta_{1}$.

Figure 3 depicts the influence of the parameters $N b$ and $N t$ on $f^{\prime}(\eta), \theta(\eta)$, and $\varphi(\eta)$ distributions. From Figure 3, it is seen that by increasing $N b$ and $N t$ parameters, the velocity inside the boundary layer decreases. Figure 3 also shows that an increase in the parameters $\mathrm{Nb}$ and $\mathrm{Nt}$ enhances the dimensionless temperature, whereas dimensionless concentration shows the same behavior, as observed in temperature distribution in the case of $\mathrm{N} t$ and the opposite behavior observed in the case of $\mathrm{Nb}$.

The graphical results for $\mathrm{Nu}$ and $\mathrm{Sh}$ are plotted against $\mathrm{Nt}$ for variation of $\mathrm{Nb}, \mathrm{Pr}$ and $\mathrm{Le}$ and shown in Figures 4 and 5. Figure 4 shows that $N u$ decreases with the increasing $N b$ while increasing with increasing $\mathrm{Pr}$ and Le. Figure 5 reveals that $\mathrm{Sh}$ rises with increasing $\mathrm{Nb}$ and $\mathrm{Le}$ and reduces with the variation of $\mathrm{Pr}$, whereas, an enhancement in this profile is more prominent in the case of $\mathrm{Nb}$ and $\mathrm{Le}$. This is due to the reason that the increase in Le is equivalent to a decrease in Brownian diffusion, and as a result, dimensionless concentration reduces and the mass transfer rate $\varphi^{\prime}(0)$ increases.

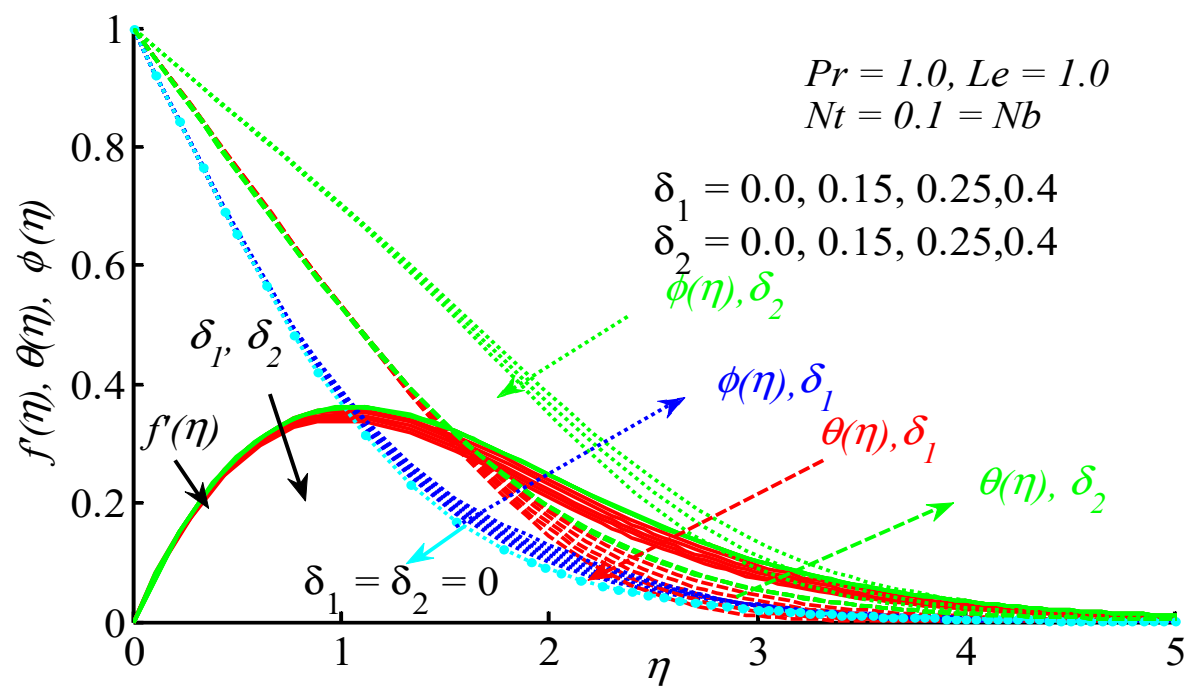

Figure 2. Effect of $\delta_{1} \& \delta_{2}$ on velocity, temperature, and concentration profiles. 


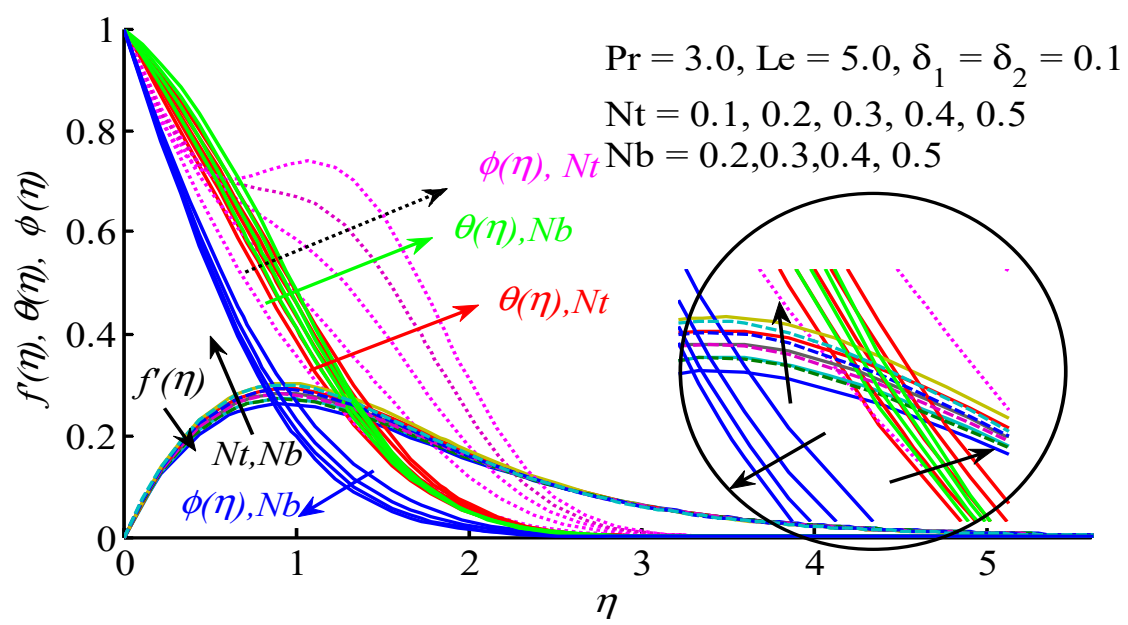

Figure 3. Velocity, temperature, and concentration distributions influenced by $\mathrm{Nt}$ and $\mathrm{Nb}$.

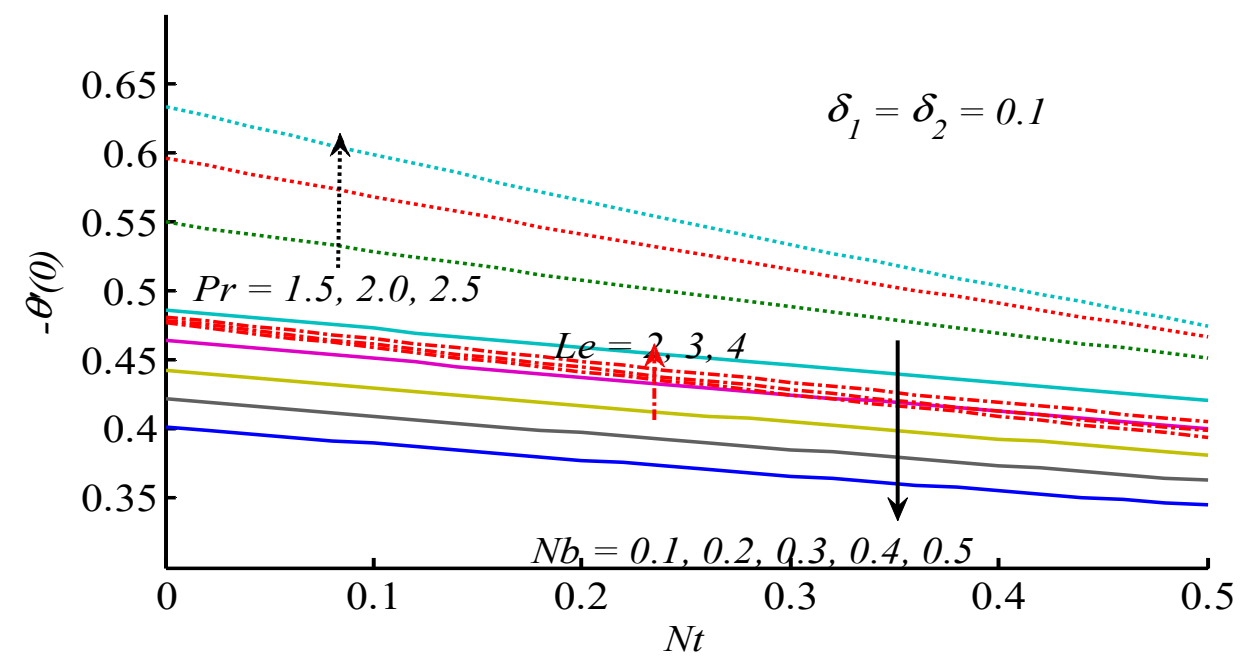

Figure 4. Influence of $\mathrm{Pr}, \mathrm{Le} \& \mathrm{Nb}$ on temperature gradient against $\mathrm{Nb}$.

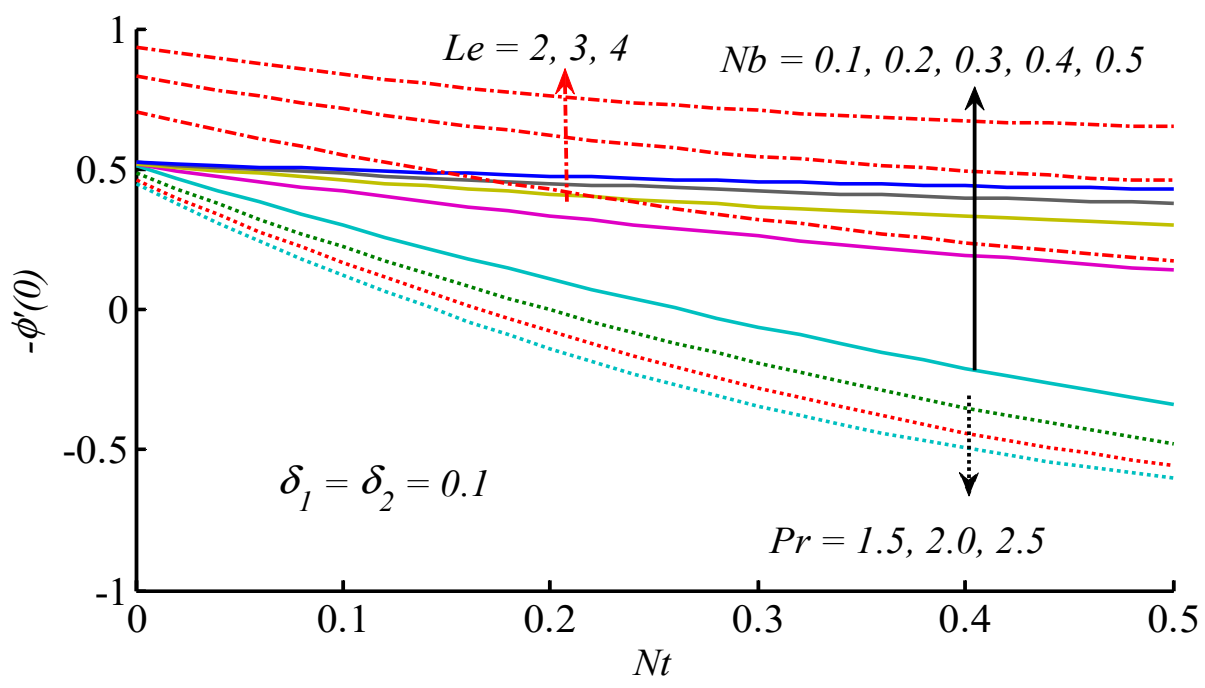

Figure 5. Variation of concentration gradient against $N t$ for different $\mathrm{Pr}, \mathrm{Le} \& \mathrm{Nb}$.

Figures 6 and 7 analyze the impact of the thermal and concentration relaxation parameters $\delta_{1}$ and $\delta_{2}$ on $N u$ and $S h$ against $N t$. Figure 6 shows that by increasing the parameters $\delta_{1}$ and $\delta_{2}, N u$ reduces and the change in the values of $N u$ is more prominent in the case of $\delta_{1}$, whereas Figure 7 
depicts that concentration of nanofluid particle at the surface increases with the increasing values of $\delta_{2}$ and opposite behavior is observed in case of $\delta_{1}$.

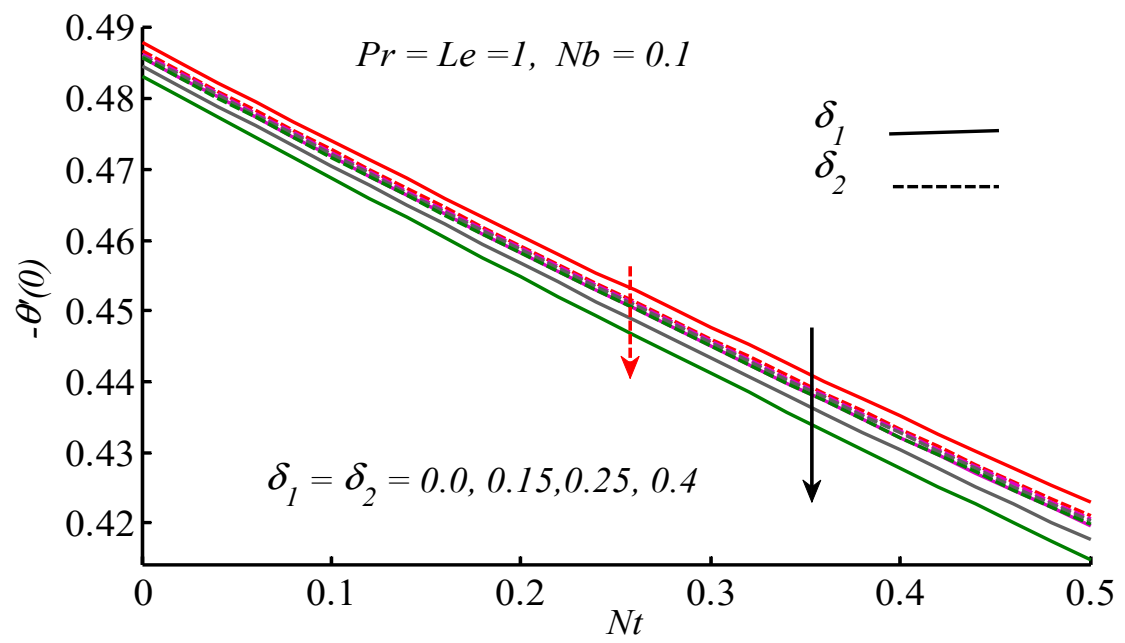

Figure 6. Variation in Nusselt number against $N t$ for different values of $\delta_{1}$ and $\delta_{2}$.

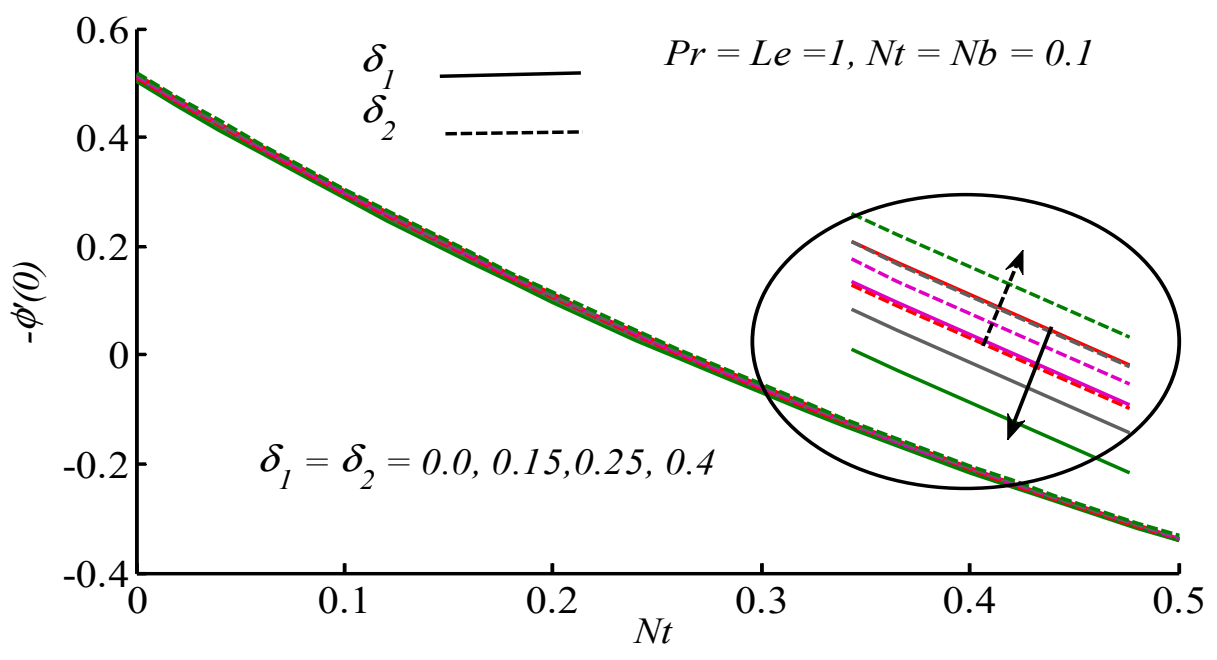

Figure 7. Sherwood number graph against $N t$ for variation of $\delta_{1}$ and $\delta_{2}$.

In Cattaneo-Christov heat and flux models, heat flux and mass flux are dependent upon the temperature gradient, concentration gradient, and the fluid velocity.

\section{Concluding Remarks}

In this paper, natural convection flow along a circular cone is investigated theoretically in the presence of Brownian motion and thermophoresis parameters of nanoparticles. Implicit finite difference technique is used to solve the transformed ordinary differential equations along with the boundary conditions. Computational results are displayed for essential quantities of physical nature such as velocity, temperature, concentration profiles, Nusselt number and Sherwood number. From this study, the obtained results are concluded here:

- With the increase of Brownian motion parameters, Sherwood number increases, whereas it gains reverse behavior against thermophoresis parameter.

- With the increase of thermophoresis and Brownian motion parameters, Nusselt number decreases.

- Nusselt number decreases by increasing Prandtl and Lewis numbers.

- Sherwood number increases by increasing Lewis numbers and decreasing Prandtl numbers. 
- Nusselt number decreases by increasing thermal and concentration relaxation parameters $\left(\delta_{1}\right.$ and $\left.\delta_{2}\right)$.

- Sherwood number increases by increasing concentration relaxation parameter $\left(\delta_{2}\right)$.

- Sherwood number decreases by increasing thermal relaxation parameter $\left(\delta_{1}\right)$.

Author Contributions: Methodology-A.G.; software-A.G.; validation, A.G., I.M.; formal analysis-I.M.; investigation, I.M.; writing-original draft preparation, M.S.I. and W.K.; writing-review and editing, W.K. and M.S.I.; funding acquisition, W.K.

Funding: This research received no external and internal funding.

Conflicts of Interest: The authors declare no conflict of interest.

\section{Nomenclature}

$\mathrm{D}_{\mathrm{T}}$

$\mathrm{D}_{\mathrm{B}}$

$\mathrm{T}$

$\alpha$

$\mathrm{g}$

$\mathrm{C}$

$N_{b}$

$N_{t}$

$T$

$T_{\infty}$

$T_{w}$

C

$C_{\infty}$

$C_{f}$

$C_{w}$

$\mathrm{Nu}$

$\mathrm{Pr}$

Sc

Sh

$\bar{u}, \bar{v}$

$u, v$

$\bar{x}, \bar{y}$

$x, y$

$r$

Gr

Le

Greek symbols

$\gamma$

$\tau$

$\phi$

$\tau_{w}$

$\psi$

$v$

$\mu$

$\rho$

$c$

$\lambda_{E}$

$\delta_{1}$

$\delta_{2}$

thermophoretic diffusion coefficient;

Brownian diffusion coefficient;

Temperature;

thermal diffusivity;

Gravitational acceleration;

Concentration;

Brownian motion parameter

thermophoresis parameter

Temperature of the fluid

Ambient fluid temperature

Surface temperature

Solutal concentration

Ambient solutal concentration

Skin friction coefficient

Solutal concentration at the wall

Nusselt number

Prandtl number

Schmidt number

Sherwood number

Dimensional velocity components in $\bar{x}$ and $\bar{y}$ directions

Dimensionless velocity components in $x$ and $y$ directions

Coordinates along and normal to the surface in dimensional form

Coordinates along and normal to the surface in dimensionless form

Radius of the base of cone

Grashof number

Lewis number

Internal half angle of the cone

The ratio of heat capacity of a nanoparticle to the base fluid

Dimensionless concentration

Wall shear stress

Stream function

Kinematic viscosity

Dynamic viscosity

Fluid density

Relaxation time of the mass flux

Relaxation time of the heat flux

Dimensionless relaxation time of the heat flux

Dimension relaxation time of the mass flux 


\section{References}

1. Choi, S.U.S. Enhancing thermal conductivity of fluids with nanoparticles. In Developments and Applications of Non-Newtonian Flows; ASME: New York, NY, USA, 1995; Volume 66, pp. 99-105.

2. Choi, S.U.S.; Lockwood, F.E.; Grulke, E.A.; Zhang, Z.G.; Yu, W. Anomalous thermal conductivity enhancement in nanotube suspensions. Appl. Phys. Lett. 2001, 79, 2252-2254. [CrossRef]

3. Hering, R.; Grosh, R. Laminar free convection from a non-isothermal cone. Int. J. Heat Mass Transf. 1962, 5 , 1059-1068. [CrossRef]

4. Hering, R. Laminar free convection from a non-isothermal cone at low Prandtl numbers. Int. J. Heat Mass Transf. 1965, 8, 1333-1337. [CrossRef]

5. Roy, S. Free convection over a slender vertical cone at high Prandtl numbers. ASME J. Heat Transf. 1974, 101, 174-176.

6. Vajravelu, K.; Nayfeh, J. Hydromagnetic convection at a cone and a wedge. Int. Commun. Heat Mass Transf. 1992, 19, 701-710. [CrossRef]

7. Kafoussias, N. Effects of mass transfer on free convective flow past a vertical isothermal cone surface. Int. J. Eng. Sci. 1992, 30, 273-281. [CrossRef]

8. Yih, K. Effect of radiation on natural convection about a truncated cone. Int. J. Heat Mass Transf. 1999, 42, 4299-4305. [CrossRef]

9. Behrang, M.A.; Ghalambaz, M.; Assareh, E.; Noghrehabadi, A.R. A new solution for natural convection of Darcian fluid about a full vertical cone embedded in porous media prescribed wall temperature by using a hybrid neural network-particle swarm optimization method. World Acad. Sci. Eng. Tech. 2011, 49, 1098-1103.

10. Cheng, C.Y. Free convection heat transfer from a non-isothermal permeable cone with suction and temperature-dependent viscosity. J. Appl. Sci. Eng. 2015, 18, 17-24.

11. Duwairi, H.M.; Zeid, O.A.; Damseh, R.A. Viscous and Joule heating effects over an isothermal cone in saturated porous media. Jordan J. Mech. Ind. Eng. 2007, 1, 113-118.

12. Elbashbeshy, E.M.A.; Emam, T.G.; Sayed, E.A. Effect of pressure work and heat generation/absorption on free convection flow from a vertical circular cone with variable surface heat flux. World J. Eng. Phys. Sci. 2013, 1, 17-25.

13. Braun, W.H.; Ostrach, S.; Heighway, J.E. Free-convection similarity flows about two dimensional and axisymmetric bodies with closed lower ends. Int. J. Heat Mass Transf. 1961, 2, 121-135. [CrossRef]

14. Grosan, T.; Postelnicu, A.; Pop, I. Free convection boundary layer over a vertical cone in a non-Newtonian fluid-saturated porous medium with internal heat generation. Tech. Mech. 2004, 24, 91-104.

15. Chamkha, A.J.; Aly, A.M.; Mansour, M.A. Effects of chemical reaction and pressure work on free convection over a stretching cone embedded in a porous medium. Int. J. Ind. Math. 2012, 4, 319-333.

16. Sohouli, A.R.; Domairry, D.; Famouri, M.; Mohsenzadeh, A. Analytical solution of natural convection of Darcian fluid about a full vertical cone embedded in porous media prescribed wall temperature through HAM. Int. Commun. Heat Mass Transf. 2008, 35, 1380-1384. [CrossRef]

17. Buongiorno, J. Convective transport in nanofluids. J. Heat Transf. 2006, 128, 240-250. [CrossRef]

18. Buongiorno, J.; Hu, W. Nanofluid coolants for advanced nuclear power plants. In Proceedings of the International Conference on Advances in Petrochemicals and Polymers, (ICAPP 05), Seoul, Korea, 15-19 May 2005; p. 5705.

19. Tiwari, R.K.; Das, M.K. Heat transfer augmentation in a two-sided lid-driven differentially heated square cavity utilizing nanofluids. Int. J. Heat Mass Transf. 2000, 50, 2002-2018. [CrossRef]

20. Mahdy, A. Natural convection boundary layer flow due to gyrotactic microorganisms about a vertical cone in porous media saturated by a nanofluid. J. Braz. Soc. Mech. Sci. Eng. 2016, 38, 67-76. [CrossRef]

21. Behseresht, A.; Noghrehabadi, A.; Ghalambaz, M. Natural-convection heat and mass transfer from a vertical cone in porous media filled with nanofluids using the practical ranges of nanofluids thermo-physical properties. Chem. Eng. Res. Des. 2014, 92, 447-452. [CrossRef]

22. Noghrehabadi, A.; Behseresht, A.; Ghalambaz, M.; Behseresht, J. Natural-Convection Flow of Nanofluids over Vertical Cone Embedded in Non-Darcy Porous Media. J. Thermophys. Heat Transf. 2013, 27, 334-341. [CrossRef] 
23. Hady, F.M.; Ibrahim, F.S.; Abdel-Gaied, S.M.; Eid, M.R. Boundary-layer flow in a porous medium of a nanofluid past a vertical cone. In An Overview of Heat Transfer Phenomena; Kazi, S.N., Ed.; IntechOpen: London, UK, 2012; pp. 91-104.

24. Fauzi, E.L.A.; Ahmad, S.; Pop, I. Mixed convection boundary layer flow from a vertical cone in a porous medium filled with a nanofluid. World Acad. Sci. Eng. Tech. 2012, 70, 10-25.

25. Khan, W.A.; Rashad, A.; Abdou, M.; Tlili, I. Natural bioconvection flow of a nanofluid containing gyrotactic microorganisms about a truncated cone. Eur. J. Mech. B/Fluids 2019, 75, 133-142. [CrossRef]

26. Khan, W.A.; Uddin, M.J.; Ismail, A.I.M. Non-similar solution of free convective flow of power law nanofluids in porous media along a vertical cone/plate with thermal and mass convective boundary conditions. Can. J. Phys. 2015, 93, 1144-1155. [CrossRef]

27. Straughan, B. Thermal convection with the Cattaneo-Christov model. Int. J. Heat Mass Transf. 2010, 53, 95-98. [CrossRef]

28. Tibullo, V.; Zampoli, V. A uniqueness result for the Cattaneo-Christov heat conduction model applied to incompressible fluids. Mech. Res. Commun. 2011, 38, 77-99. [CrossRef]

29. Kumar, K.A.; Reddy, J.R.; Sugunamma, V.; Sandeep, N. Magnetohydrodynamic Cattaneo-Christov flow past a cone and a wedge with variable heat source/sink. Alex. Eng. J. 2018, 57, 435-443. [CrossRef]

30. Han, S.; Zheng, L.; Li, C.; Zhang, X. Coupled flow and heat transfer in viscoelastic fluid with Cattaneo-Christov heat flux model. Appl. Math. Lett. 2014, 38, 87-93. [CrossRef]

31. Hayat, T.; Qayyum, S.; Imtiaz, M.; Alsaedi, A. Impact of Cattaneo-Christov Heat Flux in Jeffrey Fluid Flow with Homogeneous-Heterogeneous Reactions. PLoS ONE 2016, 11, e0148662. [CrossRef]

32. Haddad, S. Thermal instability in Brinkman porous media with Cattaneo-Christov heat flux. Int. J. Heat Mass Transf. 2014, 68, 659-668. [CrossRef]

33. Zhao, J.; Zheng, L.; Chen, X.; Zhang, X.; Liu, F. Unsteady Marangoni convection heat transfer of fractional Maxwell fluid with Cattaneo heat flux. Appl. Math. Model. 2017, 44, 497-507. [CrossRef]

34. Hayat, T.; Qayyum, S.; Imtiaz, M.; Alsaedi, A. Three-dimensional rotating flow of Jeffrey fluid for Cattaneo-Christov heat flux model. AIP Adv. 2016, 6, 025012. [CrossRef]

35. Shivakumara, I.; Ravisha, M.; Ng, C.-O.; Varun, V. A thermal non-equilibrium model with Cattaneo effect for convection in a Brinkman porous layer. Int. J. Non-Linear Mech. 2015, 71, 39-47. [CrossRef]

36. Keller, H.B. Numerical methods in boundary layer theory. Ann. Rev. Fluid Mech. 1988, 10, 793-796. [CrossRef]

37. Cebeci, T.; Bradshaw, P. Physical and Computational Aspects of Convective Heat Transfer; Springer: New York, NY, USA, 1988.

(C) 2019 by the authors. Licensee MDPI, Basel, Switzerland. This article is an open access article distributed under the terms and conditions of the Creative Commons Attribution (CC BY) license (http://creativecommons.org/licenses/by/4.0/). 